\title{
Securing Participant Engagement in Longitudinal Substance Use Disorder Recovery Research: A Qualitative Exploration of Key Retention Factors
}

\author{
Thomas Solgaard Svendsen $(\mathbb{D} \cdot$ Marius Veseth · James R. McKay • \\ Jone Bjornestad $\cdot$ Aleksander Hagen Erga $\cdot$ Christian Moltu $\cdot$ Sverre Nesvåg
}

Received: 10 February 2021 / Accepted: 28 May 2021 / Published online: 11 June 2021

(C) The Author(s) 2021

\begin{abstract}
The longitudinal cohort study is the gold standard in observational epidemiology. A central challenge with this design is the risk of attrition over time, especially in studies of inaccessible clinical populations, such as individuals with substance use disorder (SUD). Research on individuals who have achieved stable substance use abstinence and functional recovery is scarce. 30 participants from a longitudinal cohort study (the Stayer study), were interviewed concerning their experiences of
\end{abstract}

T. S. Svendsen $(\bowtie) \cdot$ A. H. Erga $\cdot$ S. Nesvåg

Centre for Alcohol and Drug Research, Stavanger

University Hospital, PO Box 8100, 4010 Stavanger,

Norway

e-mail: thla@sus.no

J. Bjornestad

Department of Social Studies, Faculty of Social Sciences,

University of Stavanger, Stavanger, Norway

J. R. McKay

Department of Psychiatry, Perelman School of Medicine, Philadelphia VA Medical Center, University of

Pennsylvania, Philadelphia, PA, USA

M. Veseth

Department of Clinical Psychology, University of Bergen,

Bergen, Norway

J. Bjornestad · C. Moltu

Department of Psychiatry, District General Hospital of

Førde, Førde, Norway participation over several years to explore retention factors. Interviewers with first-hand experience of recovery from SUD conducted the interviews. Data were analyzed using a thematic analytic approach within an interpretative-phenomenological framework.The analyses yielded the following themes: (1) Individuals' substance use: adaptation to slips and relapses in treatment and research, (2) "Show that you care": Developing working relationships in research with study participants by negotiating expectations
A. H. Erga
The Norwegian Centre for Movement Disorders,
Stavanger University Hospital, Stavanger, Norway
A. H. Erga
Department of Biological and Medical Psychology, Faculty of Psychology, University of Bergen, Bergen, Norway

\section{J. Bjornestad}
TIPS - Network for Clinical Research in Psychosis, Stavanger University Hospital, Stavanger, Norway
C. Moltu
Department of Health and Caring Sciences, Western Norway University of Applied Science, Førde, Norway


flexibly, and (3) "You don't just make dropouts": A long-term focus on study participation and treatment can increase retention. Several factors were perceived as relevant to participation in a long-term follow-up study with diverse recovery routes, including working research relationships and explicit contracts allowing follow-up participation, even during periods of drug use. Longitudinal studies could benefit from including such factors in the tracking procedures in addition to more traditional tracking techniques. Clinical services could benefit from developing interventions that use knowledge of long-term recovery processes as nonlinear, heterogeneous trajectories, and proactive approaches to motivate recovery.

Keyword Substance use disorder - Relapse · Longitudinal $\cdot$ Recovery $\cdot$ Research

\section{Background}

High retention rates in research are an indicator of a successful study with stronger internal and external validity [39, 43]. While repeated longitudinal cohort studies represent the gold standard for observational epidemiology, they are dependent on participant willingness to complete follow-ups [36].

Attrition in longitudinal studies on substance use disorder (SUD) is a widespread issue [9, 43, 56]. Therefore, focusing on effective strategies and methods to retain participants is crucial for such studies. Research on participant engagement and retention in studies finds that efforts to reduce attrition are usually described in terms of organizational strategies and tracking techniques [39]. This is also the case in studies that involve participants traditionally described as hard to reach. Examples of organizational strategies and tracking techniques are visiting practices, reminders, contact, and scheduling methods, and recruitment of trained and engaged study staff $[25,43]$. The use of motivational strategies, such as financial incentives [14] and access to study results $[29,47]$, has also been reported.

\section{Patients' Views of Study Participation and Clinical Retention Methods}

Patients' views of participation in clinical trials are gaining increasing attention, with the goals of improving processes and outcomes of care. It is common to use questionnaires, focus groups, and surveys in conducting such studies. Often, specific aspects of studies are reported, namely measures of participant preferences, evaluations of study participation, and participants' reports of health care [55]. In a recent scoping review on participant experiences of participation in clinical trials, the authors found no studies that asked participants for their feedback on the participant experience measure itself [36].

Professional SUD treatments have developed methods to meet retention challenges in clinical services [26, 32]. Therapeutic alliances and relationships between patients and clinicians are commonly considered important tools to retain patients and improve outcomes [51], and attachment style and type of substance use may also influence the association between alliances and problem reduction for individuals with SUD [17]. Continuity in care, availability of services, flexible approaches to keeping in touch, and timing of treatment episodes are often described as essential factors in SUD treatments [10, 31, 44]. A sound strategy for patient involvement seems to be key. In a systematic review of studies investigating patient preferences and shared decision making in the treatment of SUD, the authors conclude that "patients with substance use disorders should be involved in medical treatment decisions, as patients with other health conditions" ([16], p. 1).

\section{Individuals with SUD: Examples of Study Participation Barriers}

Generally low support amongst researchers for including participants who use substances in clinical studies has been reported $[28,46]$. One possible reason for this lack of support by both researchers and clinicians is the explicit or implicit stigma connected to such individuals. These forms of stigma can develop through charged language and the use of terms such as "addict" or "substance abuser" $[1,46]$, prompting a call for a people-centered language when recruiting 
for clinical studies to emphasize that substance use is a secondary attribute of the individual.

In the clinical field, self-reported feelings of guilt and self-blame amongst individuals who use substances are associated with relapse and delayed treatment seeking [30, 38]. Indeed, a number of personal vulnerabilities have been identified that can act as barriers to entering treatment. Examples are mental health challenges such as psychosis, lack of social skills, and intellectual disabilities [37]. Although utility of clinical services is not directly analogous to participation in research, we propose that barriers to participation may share several similarities.

\section{Developing Strategies to Retain Individuals with SUD in Studies}

A pitfall of longitudinal studies is the reporting of incomplete results based only on information from participants who remain. These data could differ from those from participants that have dropped out but will remain unknown because of attrition. In longitudinal statistical analyses, missing data (and dropout from studies) could greatly affect the validity of the analyses (i.e., analyses are invalidated when nonrandom data are missing) [42]. Therefore, further development of strategies to keep attrition low in long-term studies is important to ensure the validity and utility of data. One element of study retention is the potential impact of the relationships between researchers and participants. While working relationships are important in retention in substance use treatments [51], they are not commonly considered when investigating participant retention in long-term studies. Investigations of working research relationships could offer further insights into the importance of recruiting engaged research staff with knowledge of the target group [25, 39, 43].

To our knowledge, this is the first paper that investigates research relationships as a factor in participant retention in longitudinal studies of SUD. This lack of specific focus on research relationships is also found in recent studies on other groups, such as older people [15] and individuals with eating disorders [35].

\section{The Present Study}

This exploratory study is part of an ongoing longitudinal, 10-year clinical cohort follow-up study investigating long-term courses and outcomes in a sample of individuals with SUD [13, 18-21, 45, 48]. The participants in this substudy are a purposive sample of 30 individuals who met strict criteria of long-term substance abstinence and social recovery drawn from the larger project $[4,6]$. Several studies have been published based on this material, targeting participants' experiences of long-term recovery as a developmental process from dependency and reactivity to personal autonomy and self-agency $[4,6]$. The project has also investigated the effect of close relationships on recovery [53], the perceived benefits of drug use $[4,6]$, and the role of work and meaningful activities in recovery processes [54].

Representing a particularly innovative aspect of this study, the Stayer study protocol included followup interviews by people with first-hand knowledge of the phenomena of interest (i.e., addiction) as well as organizational strategies to enhance retention rates, such as reminders of appointments and flexible procedures for follow-up. In addition, we added motivational strategies, such as early establishment of working alliances, to facilitate cooperation and adapted the study protocol to the individual needs of each participant [48]. We have previously explored participants' experiences of receiving continuous feedback on their results [47], emphasizing the function of feedback and short messaging service (SMS) as important reminders of the importance of long-term efforts and comprehensive self-change in recovery processes.

The present study was to investigate key factors in staying in a longitudinal follow-up study of SUD recovery and explore helpful retention strategies when participants use substances during the study. A second aim was to investigate whether the quality of working relationships in the study influenced retention in the study and whether this knowledge had useful implications for clinical SUD services.

\section{Methods}

We used a thematic analytic approach [3, 49] developed within an interpretative-phenomenological 
framework [40, 52]. We developed objectives and procedures within a user-involved research framework $[2,24]$. We recruited two service users with first-hand knowledge of long-term recovery from SUD. They contributed to developing the interview guide, conducting the interviews, analyzing the data, and reporting on the study. The collaborative aspects and service user involvement in this study are explained by Veseth et al. [53].

\section{Sample and Recruitment}

The sample was recruited from the ongoing Stayer study ( $\mathrm{n}=202)$, a 10-year, naturalistic follow-up study of change trajectories following SUD in Rogaland, Norway. Participants were included between March 2012 and December 2015 from outpatient and residential treatment facilities at the start of treatment. Inclusion criteria were starting a new treatment sequence, diagnosis with SUD, and being $\geq 16$ years of age. Retention rates in the study were $91 \%$ at the 12-month assessment and $70 \%$ at the 72-month assessment. We recruited substudy participants for consecutive interviews at their four- or five-year follow-ups. The Stayer study team conducted a screening process based on objective criteria for stable substance abstinence and social recovery (see Measures).

\section{The Stayer Study Protocol}

We used biweekly SMS tracking to gather data on the consumption levels of participants and their contact with treatment services. Biweekly monitoring was used to find the optimal balance between gathering real-time data while not overburdening participants by using an excessively demanding protocol.

The baseline assessment in the study used 16 instruments and self-report forms. Quarterly assessments were conducted in the first 24 months with eight instruments and self-report forms. The annual assessment used 14 instruments and self-report forms (see "Appendix"). We used organizational and motivational strategies to enhance retention rates in the Stayer study, such as reminders of appointments, flexible visiting characteristics [48], and access to data on request [47].
Measures

We used the following instruments in this study: (1) the Drug Use Disorders Identification Test (DUDITC) to assess drug use [2], (2) the Alcohol Use Disorders Identification Test (AUDIT-C) to assess alcohol consumption, (3) the Symptom Checklist 90 Revised (SCL-90-R) to assess psychological functioning [11] based on the summarized Global Severity Index (GSI), (4) the Behavior Rating Inventory of Executive Functions-Adult Version (BRIEF-A) to assess executive functioning [41], and (5) the Satisfaction With Life Scale (SWLS) to assess quality of life [12]. Substance abstinence was defined as a DUDIT-C score of 0 and AUDIT-C scores $<2$. Relapse was defined as a score above the cut-off for either alcohol or drug use during the previous two years. Social functioning was defined using four variables related to social functioning status: housing, income, friends without addiction, and participation in work or school. Participants who met the criteria for positive functioning status on all four social variables were categorized as having adequate social functioning. Recovery was defined as meeting the criteria for both stable substance abstinence and adequate social functioning in the previous two years.

\section{Interviews}

Interviews were conducted between October 2017 and April 2018 by two long-term recovered service users, with two pilot interviews conducted prior to the study. We developed a semi-structured interview guide in line following the recommendations of Miles, Huberman, and Saldaña [33] based on reports of factors facilitating SUD recovery (e.g., [22, 31, 34, 52]. The following focus areas guided the interview: (1) personspecific factors, (2) environmental factors, (3) treatment-related factors, and (4) experiences of participation in the Stayer study. Each theme was introduced with an open-ended question such as: "How would you describe your experiences of participation in the Stayer study?" We used follow-up questions that encouraged participants to relate their experiences, for example, asking "Could you elaborate on your experiences of remaining in the Stayer study for several years?" To address topics not adequately covered by the interview, participants were invited at 
the end of each session to provide any further relevant information.

Interviews were conducted by two clinically recovered service users who received training in semistructured interviewing by J.B. The interviews provided a dataset developed through semi-structured conversations between peers [22, 53]. Interviews (mean duration, $57 \mathrm{~min}$,range, 27-96 min) were conducted at Stavanger University Hospital $(\mathrm{n}=25)$, a participant's home $(n=1)$, and by telephone $(n=4)$. Interviews were audio recorded and transcribed verbatim for analysis.

\section{Analysis}

Our analysis employed the seven-step procedure of thematic analysis [8], outlined in Table 1. To strengthen the credibility of the study, four of the researchers (J.B., T.S.S., M.V., and C.M.) [6] conducted the entire procedure independently. The same researchers met to compare their interpretations, agreed on themes with accompanying quotes, and validated the findings by consensus [23], dedicating special attention to steps four to seven, shown in Table 1. Because the present study in a sense is metaresearch, it is an exploration of participants' experiences of research. We involved researchers in the analysis phase (M. V. and C. M.) who were not directly involved in following up the Stayer study participants on a day-to-day basis, to provide outsidein views on the data material.
Ethics

The Regional Ethics Committee in Norway (2011/ 1877) approved the study. Ethical issues were discussed throughout the research process, from planning process to publication. We obtained written informed consent from all participants prior to the study. We took care in the interviews and in working with the material to treat participants' experiences with respect [53].

\section{Results}

Demographic, clinical, treatment, psychological, and social variables are shown in Table 2 . In presenting the results, we refer to 20-30 participants as "most," 10-19 as "many," and 5-9 as "some" of the participants [23]. Participants described essential factors for continuous participation in a long-term study. Three sub-themes are: (1) participants' reflections on the effects of substance use on research and treatment participation, (2) the importance of working relationships in continuous study and treatment participation, and (3) improvement of retention in treatment and research caused by a long-term focus.

Table 1 Steps of text condensation

1 Becoming familiar with the data through thorough reading of the transcribed interviews, forming a main impression of the experiences of the participants, and identification of potential important themes. A theme was defined as a verbalization capturing an important element of the data in relation to the research question, representing a patterned response in the data set

2 Generating initial codes, which were defined as the most basic segments of the raw data that could be assessed in a meaningful way regarding the phenomenon

3 Searching for and developing candidate themes and sub-themes. Remaining codes were set aside at this phase in a separate category for the purpose of being further analyzed and incorporated when appropriate

4 Reviewing themes to develop a coherent thematic map and considering the validity of individual themes in relation to the data set

5 Defining and naming themes: Further refining and defining themes, identifying the essence of themes, identifying subthemes and summarizing the contents of the main themes into what each researcher considered to best represent participants' experiences. When our refinements no longer added substantially to the themes, the analytic process was closed

6 To determine the relevance of a particular theme we both counted the frequency of the relevant meaning units combined with our interpretation of how central the theme was perceived to the recovery process

7 Last, the tentative model of findings, with illustrative quotes, was sent to two fully recovered service users who served as critical auditors assessing the interpretations made through our descriptions of the central organizing concepts 
Table 2 Baseline and follow up demographic, clinical, treatment-related, psychological and social variables

\begin{tabular}{|c|c|c|c|c|c|c|}
\hline & \multirow{2}{*}{$\begin{array}{l}\text { Baseline } \\
(\mathrm{N}=30)\end{array}$} & \multirow{2}{*}{$\begin{array}{l}\text { Year } 1 \\
(\mathrm{~N}=30)\end{array}$} & \multirow{2}{*}{$\begin{array}{l}\text { Year } 2 \\
(\mathrm{~N}=30)\end{array}$} & \multirow{2}{*}{$\begin{array}{l}\text { Year } 3 \\
(\mathrm{~N}=30)\end{array}$} & \multicolumn{2}{|c|}{ Endpoint assessment } \\
\hline & & & & & $\begin{array}{l}\text { Year } 4 \\
(\mathrm{~N}=10)\end{array}$ & $\begin{array}{l}\text { Year } 5 \\
(\mathrm{~N}=20)\end{array}$ \\
\hline \multicolumn{7}{|l|}{ Demographics } \\
\hline Age & $25.9(5.5)$ & & & & & \\
\hline Male/female, $n$ & $17 / 13$ & & & & & \\
\hline Education, years & $12.8(1.8)$ & & & & & \\
\hline \multicolumn{7}{|l|}{ Substance use history } \\
\hline Age of initial use & $13.1(1.8)$ & & & & & \\
\hline Years of drug use & $12.9(6.0)$ & & & & & \\
\hline AUDIT score & $11.9(11.4)$ & $3.4(7.6)$ & $2.3(4.1)$ & $2.9(6.8)$ & $4.4(7.0)$ & $2.2(3.2)$ \\
\hline DUDIT score & $29.0(15.9)$ & $6.6(13.1)$ & $3.1(11.5)$ & $1.9(8.5)$ & $0(-)$ & $0(-)$ \\
\hline \multicolumn{7}{|l|}{ Treatment } \\
\hline Previous treatment attempts & $1.3(2.0)$ & - & - & - & - & - \\
\hline Currently outpatient, n (\%) & $13(43.3)$ & $17(56.7)$ & $8(26.7)$ & $5(16.7)$ & $2(20.0)$ & $2(9.5)$ \\
\hline Currently inpatient, n (\%) & $17(56.7)$ & $5(16.7)$ & $4(13.3)$ & $2(6.7)$ & $0(0)$ & $0(0)$ \\
\hline $\begin{array}{l}\text { Currently in self-help group }{ }^{\mathrm{a}}, \mathrm{n} \\
(\%)\end{array}$ & $13(43.3)$ & $13(43.4)$ & $15(50.0)$ & $10(33.3)$ & $4(40.0)$ & $3(14.3)$ \\
\hline \multicolumn{7}{|l|}{ Social variables ${ }^{b}$} \\
\hline Permanent housing, n (\%) & $15(50.0)$ & $25(83.3)$ & $25(83.3)$ & $26(86.6)$ & $10(100)$ & $21(100)$ \\
\hline Stable income, n (\%) & $16(53.3)$ & $21(70.0)$ & $27(90.0)$ & $27(90.0)$ & $10(100)$ & $21(100)$ \\
\hline Employed/student, n (\%) & $5(16.7)$ & $7(23.3)$ & $14(46.7)$ & $19(63.3)$ & $10(100)$ & $21(100)$ \\
\hline Abstinent friends ${ }^{\mathrm{c}}, \mathrm{n}(\%)$ & $24(80.0)$ & $25(83.3)$ & $26(86.7)$ & $27(90.0)$ & $10(100)$ & $21(100)$ \\
\hline \multicolumn{7}{|l|}{ Psychological measures } \\
\hline SCL90-R GSI & $1.2(0.7)$ & $0.7(0.7)$ & $0.6(0.5)$ & $0.5(0.4)$ & $0.5(0.4)$ & $0.4(0.5)$ \\
\hline BRIEF-A GEC & $67.2(11.3)$ & $57.2(11.3)$ & $54.9(12.6)$ & 51. (10.9) & $52.5(10.5)$ & $50.4(11.2)$ \\
\hline SWLS, sum score & $17.5(6.8)$ & $24.8(6.7)$ & $24.8(5.2)$ & $25.2(5.4)$ & $25.3(2.7)$ & $27.4(5.0)$ \\
\hline
\end{tabular}

All numbers are mean (SD), unless otherwise specified. Abbreviations: SCL-90-R GSI symptom checklist 90 revised global severity index T-score, BRIEF-A GEC behavioral rating inventory of executive function adult version global executive composite T-score, $S W L S$ satisfaction with life scale, AUDIT alcohol use disorders identification test, DUDIT drug use disorder identification test

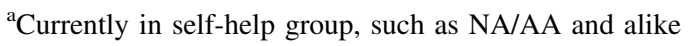

${ }^{\mathrm{b}}$ Social variables are positive responses to yes/no questions

${ }^{\mathrm{c}}$ Friends without a history of substance use

Thematic Analysis

\section{Individuals' Substance Use: Adaptation to Slips and Relapses in Treatment and Research}

In the early stages of the Stayer study, many participants used several substances and lived in unstable life conditions. The study's retention strategies needed to take this into consideration when following up with participants. Their change trajectories developed in a nonlinear fashion over time, with slips and relapses.
Many participants reported that periodic substance use complicated their efforts to continue with treatment, leading to gaps and dropouts. At the same time, experiences of slips and relapses were used as springboards in their individual initiatives in treatment, because these experiences clarified that desired life changes were difficult to achieve alone.

Yeah, I went into treatment. Then I was back and forth there several times before I became truly sober. You were at the bottom, right, it was just 
fucking...there was nothing left of the substance using life. It was just gibberish, you know. So I went into treatment, danced around a few times, had a few slips, went into treatment again, went to jail, and then, the last time there [in jail] I thought: "Fuck, I need to lie down flat, sort of. I am not going to give a damn about some of the things I feel are important, I'm going to leave them for dead. I'm going to listen to what people tell me to do. I'm going to try that, sort of. And then it worked. Slowly but surely, the changes happened, and I started to figure out that I could do it. (Participant 1044).

During these periods of substance use slips and relapses, many participants also described periodical difficulties remembering appointments and following the Stayer study protocol of regular assessments. Researchers' deductions about participants' reported substance use symptoms based on their personalities were considered important for continuous study participation, even when participants arrived for assessments while under the influence.

Participant 1006: When I was using, if I wasn't able to answer these questions and I forgot appointments, then it wasn't held against you in the future, and that is important I think. To remember that we don't choose to be assholes, it is just how it is.

Interviewer: And that you can show up high? Participant 1006: Yes.

Accepting the study participants' life experiences, their periodic substance use, and avoiding dichotomous statements about how they were perceived, were considered important for continued study participation. In avoiding statements about right and wrong, many participants reported that they could speak honestly about their present life situations and be more themselves.

[...] sorts of accepts what I do in my life, and not...sort of. There is no preaching about right and wrong. And it was very good for me, that I felt that I could be more like myself. Then I can say what I actually mean, and I don't get a response about me being wrong [...]. If I had to be clean to participate, and be taught what is good for me and stuff like that, then I don't think that I would have continued participation. (Participant 1032).

The Stayer study's long-term perspective on substance use disorder recovery processes meant that participants were not excluded from the study if they were unable to complete follow-ups. Participants could continue using substances, and study staff were not tasked with motivating participants specifically to stop using substances. This was perceived as important by many participants.

I don't know, it is a long term follow up. And the fact that many of the participants still use and continue to participate, that is great. That you don't just make drop outs out of people that are using. (Participant 1033).

The Stayer study staff attempted to stay in touch with participants over time, regardless of substance use or mental health situations. These nonlinear change trajectories required differentiated strategies to stay in touch with the participants. Many participants described their experiences of being unable to follow the study protocol successfully, while still being approached by study staff to keep in touch, as one of the reasons for remaining in the study.

What I think is positive is that it is very hard to stay in touch with people. And I believe that to be a bit more proactive... because I feel that in many treatments it's like black or white, like if you relapse it's like: “Ok, you are out". And I don't think that works. Because most people don't experience sort of: "Ok, now I'll stop. Now I am finished". Not many people experience that. To understand that it takes time to quit. And that people sort of go through a process that is up and down, and to still keep in touch, and be able to come back. So that proactive...that [...] is proactive and outreaching and tries to get in touch. (Participant 1137).

\section{"Show That You Care": Developing Working Relationships in Research with Study Participants by Negotiating Expectations Flexibly}

Most participants in the Stayer study had several attempts to change their substance use and treatment episodes behind them, and many made several 
attempts at change in the early phases of the study. Thus, retention strategies needed to be tailored to individual needs in different periods of their lives. Many participants expressed clear opinions about treatment services that they had received during previous change attempts and how they could be developed. Suggested changes were individualizing clinical skills and showing genuine empathy for the person needing treatment.

To care about what the person that is sitting in the chair actually needs. Not just follow routines and go by the book, but actually see every person. Because we don't need exactly the same things; we are different. I need one thing, you need another. And to actually care. I get pissed when people are just sitting there to have a job. "No, you can't call after office hours"... show that you care! And that you actually are the most important person. It's not them, it's you. (Participant 1032).

Many participants compared their experiences of ongoing participation in the Stayer study with treatment relationships outside the research frames. Many participants described the study's emphasis on prioritizing their needs, even when they were using substances, as important. Research staff members' comments about participants' normal and expected accountability to continue in the study was highlighted.

And someone who cares to spend...yeah, spend time on you even though... and want you to come even when you are...it is very important, when everyone else doesn't even want to see you, and then: "Hi, are you coming to our meeting today?" like why shouldn't you? It is amazingly nice when someone expresses that they expect something from you, when no one is expecting anything from you or wants to bring you along for things. (Participant 1006).

Establishing a working relationship between researchers and participants led to many participants expressing an obligation to make their appointments, even during periods of substance use or poor mental health. The early establishment of safe working relationships based on trust was described as an important factor in continued participation by many participants.
I think it has been very nice, or rewarding. It is a reason why I have always followed up, and it says quite a lot about our relationship, that we established it quite fast. That led to me caring about meeting, or else I wouldn't have done it. Even though I found it interesting [the Stayer study]. But when I showed up and was in many different states, then I wasn't all that interested in it [the study]. Then it was because I knew that [...] was a safe person for me, so that meant a lot. (Participant 1006).

\section{"You Don't Just Make Dropouts": Long-Term Focus on Study Participation and Treatment Can Lead to Higher Retention}

Many participants reflected on how slips and relapses did not necessarily lead to treatment discharge during early stages of the study. Experiences of not being abandoned by the treatment team were seen as important to continue working for a life not dominated by substance use. Many participants also perceived that periodic substance use while in treatment or between treatments offered experiences that motivated changes in substance use.

Participant 1148: And when I went into treatment, then it's like, if you slip they don't give up on you. You can come back. And I had several slips in there, and I got to come back every time. So that's a full package that helped me there. For every slip I had, I learned something. And I learned to come back, and I developed a strength within myself to want and to manage to be sober, sort of.

Interviewer: Yeah, and it is great that you didn't give up?

Participant 1148: Yeah, and that they did not give up on me.

Interviewer: That's true.

Participant 1148: I've been to many treatments. I have always used [substances] in the treatment, and then left. And they give up on you fast, sort of. To have a place you can come back to, and start fresh. Or, from where you are. They understand that, and me.

Many participants' feelings of self-blame in periods of substance use were described as a barrier to 
continued Stayer study participation. The fact that the Stayer study was designed to maintain contact with participants, with a commitment to regular appointments whenever possible, was described as important for retention. Many participants also expressed the view that more people would stay in touch with treatment services if expressions of individual guilt and shame were avoided.

Yeah, and not just give up if someone isn't answering their phone or doesn't show up for an appointment. Because it's so much shame and guilt and feelings like that, and if you feel: "Ok, I fucked up," so now it's my own fault and now I have to continue living like this...if you could skip that part, then I think that could help a lot of people. (Participant 1033).

Expectations of punishment and intimidation when participants showed up for appointments when they were not subjectively feeling their best was described as a potential barrier to continued study participation. Many participants reported reluctance to partake in the study during troublesome periods in their lives.

Because people are at so different places in their lives when they meet [...]. It can't be intimidating, because then I think a lot of people would have dropped out. They would be afraid of punishment, and like "now I am not doing so well, so I should keep away from there”, right? (Participant 1137).

The Stayer study staff members consistently reminded participants that substance use recovery is often a long-term process, and that flexibility in making appointments was an important part of the study design. Many participants reported that this flexibility made it easier to stay in the study over time.

I think it has been great [to participate in Stayer]. One thing is that you are part of something. But as a rule you participate for a short period of time, and then you drop out, right? But for some reason that hasn't happened here. And I think that some of the reason for that is that I don't have to see you at a specific time [...] sort of make it up to you, so that it is easy for me. (Participant 1033).

Flexibility in making appointments for patients in treatment, and reflections on normal, expected accountability by research staff were also highlighted regardless of substance use status. Some participants considered that clinical services could benefit from proactive methods and accountability for follow-up treatment appointments.

Say that you are at the psychologist's office, then you can choose not to show up. But if the psychologist is a bit proactive ... my psychologist was like that if I was a bit late: "You are coming today? You are, right? We have an appointment today, you know?" "Yeah, I'm just a bit late." (Participant 1137).

\section{Discussion}

Our findings suggest that longitudinal follow-up study participation by individuals seeking to eliminate or reduce substance use requires engaging working relationships with research staff and positive attitudes of researchers toward participants. Accepting participants' life experiences with periodic substance use, avoiding dichotomous pejorative statements, and recognizing that SUD recovery often entails longterm nonlinear processes were perceived as facilitating retention. Moreover, these same factors emerged from participants' descriptions of successful treatment leading to long-term abstinence.

During periods of substance uses in the early stages, participants were often admitted to outpatient or residential treatment, and they reflected that factors such as empathy and individualized treatment could have improved their treatment processes and outcomes. This is consistent with studies showing that recovery management and continuing care provided through ongoing monitoring and early re-intervention approaches may sustain recovery from chronic SUDs and reduce slips and relapses [10, 44]. Our findings suggest that longitudinal follow-up studies, using methods such as a relational focus between researchers and participants, as well as positive attitudes toward participants, can facilitate long-term retention in longitudinal studies.

Implications for Research

Longitudinal, prospective follow-up studies on SUD are in a unique position to develop scientific 
knowledge about change processes that cannot be identified clearly in short-term studies or in those without follow-up. For example, a recent review investigated how often outcomes other than change in substance use are measured in RCTs. The review found that $42.1 \%$ of 504 studies followed participants for 13 weeks or less, and that only $3.8 \%$ followed participants for two years or more [5]. To identify the key factors in recovery from SUD, more longitudinal studies are needed that include strategies for adaptation to slips and relapses and facilitate working relationships between researchers and participants by negotiating expectations and participants' accountability flexibly to maintain high retention.

Our findings indicate that working research relationships and researchers' positive attitudes towards participants were important for remaining in the Stayer study. Moreover, many participants highlighted that accountability for attempting to adhere to the study protocol was an important factor in their retention. It is not a one-way street, with all expectations on the participants. Researchers in long-term studies have a responsibility to express nonjudgmental attitudes towards participants if they expect follow-up in return. These factors are relevant to clinical service delivery, considering participants' expressions of selfblame, guilt, shame [30,38], and feelings that they fall short of society's expectations when using substances $[28,46]$. The consequences can be treatment intermissions, dropout, and lack of early interventions when slips and relapses occur.

Our second theme, "Show that you care," is related to a growing literature on recovery-oriented professionals. For example, Borg and Kristiansen [7] emphasize the importance of professionals being willing to stretch the boundaries of the traditional professional role. Topor, Skogens, and Greiff [50] state that clinicians must be accepted as trustworthy agents in motivating change for individuals with cooccurring addiction and mental health problems. Our study findings support this view and underscore how this may be equally important in research on people with SUD.

\section{Implications for Clinical Service Delivery}

In SUD treatments, successful strategies include strong, therapeutic alliances, expressions of hope that individuals can stop using substances, empathizing, and individualization [10, 31, 44]. A key point regarding individualized treatments is the individual's substance use itself. Our findings indicate that care should be taken in treatment relations to avoid exposing individuals to clinicians' potential negative and moralizing statements during treatment. This is not to say that expressing hope and establishing relational alliances should be avoided. It could suggest that when experiencing guilt and shame, individuals benefit from meeting clinicians who know that longterm changes often require long-term effort and nonlinear, heterogeneous trajectories. Treatment efforts could be timed better, without fixed guidelines for life changes, including substance use. Although this observation resonates well with essential therapeutic skills in psychotherapy or milieu therapy for SUD, we argue that these findings also generalize to other interventions in the SUD field, including treatment schedules in correctional facilities, individual placement and support training, occupational training, and social skills training.

This focus on timing of treatments and research should be combined with normal accountability for individuals following up treatment, to avoid removing all personal responsibility from the individual and ensure involvement from all parties. The findings in the present study suggest that SUD treatments and research studies are more likely to succeed when welltimed, proactive efforts are used with the same expectations of regular contact as for any other people in clinical treatment [27]. Emphasizing cooperation between service providers in substance treatments and follow-up services could offer proactive solutions and better-timed, interdisciplinary services that are often required when individuals seek to make comprehensive life changes.

\section{Limitations}

First, our findings are context dependent, as the participants were recruited from outpatient and residential treatment in the region of Stavanger, Norway, possibly affecting transferability to other contexts. Second, participants shared their retrospective reflections on study participation and on periods of slips and relapse. Reflections on life events and processes in earlier times could be affected by natural personal development and life events, as well as lapses in memory. This is a general, possible limitation in all 
qualitative studies investigating retrospective events. Third, we recruited participants with good functioning levels at the time of the interviews, and a high percentage had also had good functioning levels prior to experiencing SUD. This is not to suggest that these participants were not at risk of long-term functional disability. This is clarified in the present study by participants' individual descriptions of comprehensive treatment efforts and several substance use slips and relapses over years of participation, eventually to manage long-term abstinence from substance use. Finally, awareness of our own research practices is called for in our study of participants' views of participation, and it was necessary to involve researchers that were not directly involved in following up the Stayer study participants on a day-to-daybasis. M. V. and C. M. were, therefore, first involved in the analysis phase of the study to ensure the inclusion of outside-in views on the data material.

\section{Conclusions}

Several aspects of research relations were perceived to be relevant to extended participation in a long-term follow-up study on diverse recovery routes. Longitudinal study designs could benefit from including discussions on research relations, management of participant retention, even in periods of substance use slips and relapses, and researchers' potential prejudice toward individuals with SUD to retain participants. Clinical services could benefit from developing services that use knowledge of SUD recovery processes, involving nonlinear, heterogeneous trajectories, proactive approaches to keeping individuals in treatment, and standard agreements on accountability between individuals and clinicians to motivate recovery.

\begin{abstract}
Acknowledgements This study could not have been performed without the effort and time of the participants. We thank the interviewers in this study, Tale Ekeroth Slyngstad and Aleksander Waagan Skaalevik, for their solid contributions and their use of lived experiences in the research process to produce rich data. Geir Strandenaes Larsen, Academic Librarian in Stavanger University Library, Norway, was an important resource in conducting systematic literature searches.
\end{abstract}

Open Access This article is licensed under a Creative Commons Attribution 4.0 International License, which permits use, sharing, adaptation, distribution and reproduction in any medium or format, as long as you give appropriate credit to the original author(s) and the source, provide a link to the Creative Commons licence, and indicate if changes were made. The images or other third party material in this article are included in the article's Creative Commons licence, unless indicated otherwise in a credit line to the material. If material is not included in the article's Creative Commons licence and your intended use is not permitted by statutory regulation or exceeds the permitted use, you will need to obtain permission directly from the copyright holder. To view a copy of this licence, visit http://creativecommons.org/licenses/by/4.0/.

\section{Appendix}

\section{Measures of neurocognitive and psychosocial functioning}

Regional quality register for treatment of addiction. Alcohol Use Disorder Identification Test (AUDIT). Drug Use Disorder Identification Test (DUDIT). The Symptom Checklist 90 Revised (SCL-90-R). Snaith-Hamilton Pleasure Scale (SHAPS).

Satisfaction With Life Scale (SWLS).

Pittsburgh Sleep Quality Index (PSQI).

The Montreal Cognitive Assessment (MOCA).

Wechsler Abbreviated Scale of Intelligence (WASI).

Iowa Gambling Task (IGT).

Stroop.

Behaviour Rating Inventory of Executive Function-Adult Version (BRIEF-A).

Trail Making TEST (TMT) Parts A and B.

Conner's Continuous Performance Test II Version 5 (u II V.5).

Adult ADHD Self-Report Scale (ASRS-v1.1).

Revised NEO Personality Inventory (NEO PI-R).

\section{References}

1. Ashford RD, Brown AM, Curtis B. Substance use, recovery, and linguistics: the impact of word choice on explicit and implicit bias. Drug Alcohol Depend. 2018;189:131-8.

2. Berman AH, Bergman H, Palmstierna T, Schlyter F. Evaluation of the drug use disorders identification test (DUDIT) in criminal justice and detoxification settings and in a 
Swedish population sample. Eur Addict Res. 2005;11(1):22-31.

3. Binder P-E, Holgersen H, Moltu C. Staying close and reflexive: an explorative and reflexive approach to qualitative research on psychotherapy. Nord Psychol. 2012;64(2):103-17.

4. Bjornestad B, Veseth M, Berg H, Davidson L, McKay JR, Moltu C, Skaalevik AW, Slyngstad TE, Svendsen TS, Nesvåg S. Reports of the benefits of drug use from individuals with substance use disorders. Psychother Res. 2019;30(6):718-27.

5. Bjornestad J, McKay JR, Berg H, Moltu C, Nesvåg S. How often are outcomes other than change in substance use measured? A systematic review of outcome measures in contemporary randomised controlled trials. Drug Alcohol Rev. 2020;39(4):394-414.

6. Bjornestad J, Svendsen TS, Slyngstad TE, Erga AH, McKay JR, Nesvåg S, Skaalevik AW, Veseth M, Moltu C. "A life more ordinary" processes of 5-year of recovery from substance abuse. Experiences of 30 recovered service users. Front Psychiatry. 2019;10:689.

7. Borg M, Kristiansen K. Recovery-oriented professionals: helping relationships in mental health services. J Ment Health. 2004;13(5):493-505.

8. Braun V, Clarke V. Using thematic analysis in psychology. Qual Res Psychol. 2006;3(2):77-101.

9. Claus RE, Kindleberger LR, Dugan MC. Predictors of attrition in a longitudinal study of substance abusers. J Psychoact Drugs. 2002;34(1):69-74.

10. Dennis ML, Scott CK, Laudet A. Beyond bricks and mortar: recent research on substance use disorder recovery management. Curr Psychiatry Rep. 2014;16:442.

11. Derogatis LR. SCL-90-R administration, scoring, and procedures manual II for the revised version. 2nd ed. Towson, MD: Clinical Psychometrics Research; 1983.

12. Diener E, Emmons RA, Larsen RJ, Griffin S. The satisfaction with life scale. J Pers Assess. 1985;49(1):71-5.

13. Erga AH, Hønsi A, Anda-Ågotnes LG, Nesvåg S, Hesse M, Hagen E. Trajectories of psychological distress during recovery from polysubstance use disorder. Addict Res Theory. 2020. https://doi.org/10.1080/16066359.2020. 1730822.

14. Festinger DS, Marlowe DB, Dugosh KL, Croft JR, Arabia PL. Higher magnitude cash payments improve research follow-up rates without increasing drug use or perceived coercion. Drug Alcohol Depend. 2008;96(1-2):128-35.

15. Forsat ND, Palmowski A, Palmowski Y, Boers M, Buttgereit F. Recruitment and retention of older people in clinical research: a systematic literature review. J Am Geriatr Soc. 2020;68(12):2955-63.

16. Friedrichs A, Spies M, Härter M, Buchholz A. Patient preferences and shared decision making in the treatment of substance use disorders: a systematic review of the literature. PLoS ONE. 2016;11(1):e0145817.

17. Gidhagen Y, Holmqvist R, Philips B, Falkenström F. The role of the working alliance in psychological treatment of substance use disorder outpatients. Psychother Res. 2020. https://doi.org/10.1080/10503307.2020.1807639.

18. Hagen E, Erga AH, Hagen KP, Nesvåg SM, McKay JR, Lundervold AJ, Walderhaug E. Assessment of executive function in patients with substance use disorder: a comparison of inventory- and performance-based assessment. J Subst Abuse Treat. 2016;66:1-8.

19. Hagen E, Erga AH, Hagen KP, Nesvåg SM, McKay JR, Lundervold AJ, Walderhaug E. One-year sobriety improves satisfaction with life, executive functions and psychological distress among patients with polysubstance use disorder. J Subst Abuse Treat. 2017;76:81-7.

20. Hagen E, Erga AH, Nesvåg SM, McKay JR, Lundervold AJ, Walderhaug E. One-year abstinence improves ADHD symptoms among patients with polysubstance use disorder. Addict Behav Rep. 2017;6:96-101.

21. Hagen E, Sømhovd M, Hesse M, Arnevik AE, Erga HA. Measuring cognitive impairment in young adults with polysubstance use disorder with MoCA or BRIEF-A-The significance of psychiatric symptoms. J Subst Abuse Treat. 2019;97:21-7.

22. Harris M. "Three in the room": embodiment, disclosure, and vulnerability in qualitative research. Qual Health Res. 2015;25(12):1689-99.

23. Hill CE, Knox S, Thompson BJ, Williams EN, Hess SA, Ladany N. Consensual qualitative research: an update. J Couns Psychol. 2005;52(2):196.

24. Hill CE, Thompson BJ, Williams EN. A guide to conducting consensual qualitative research. Couns Psychol. 1997;25(4):517-72.

25. Kleschinsky JH, Bosworth LB, Nelson SE, Walsh EK, Shaffer HJ. Persistence pays off: follow-up methods for difficult-to-track longitudinal samples. J Stud Alcohol Drugs. 2009;70(5):751-61.

26. Knuuttila V, Kuuisto K, Saarnio P, Nummi T. Effect of early working alliance on retention in outpatient substance abuse treatment. Couns Psychol Q. 2012;25(4):361-75.

27. Laferton JAC, Kube T, Salzmann S, Auer CJ, SheddenMora MC. Patients' expectations regarding medical treatment: a critical review of concepts and their assessment. Front Psychol. 2017;8:233.

28. Leeman RF, Hefner K, Frohe T, Murray A, Rosenheck RA, Watts BV, Sofuoglu M. Exclusion of participants based on substance use status: findings from randomized controlled trials of treatments for PTSD. Behav Res Ther. 2017;89:33-40.

29. Long CR, Stewart MK, McElfish PA. Health research participants are not receiving research results: a collaborative solution is needed. Trials. 2017;18(1):449.

30. Luoma JB, Kohlenberg BS, Hayes SC, Fletcher L. Slow and steady wins the race: a randomized clinical trial of acceptance and commitment therapy targeting shame in substance use disorders. J Consult Clin Psychol. 2012;80(1):43-53.

31. McKay JR. Treating substance use disorders with adaptive continuing care. Washington, DC: American Psychological Association; 2009.

32. Meier PS, Barrowclough C, Donmall MC. The role of the therapeutic alliance in the treatment of substance misuse: a critical review of the literature. Addiction. 2005;100(3):304-16.

33. Miles MB, Huberman AM, Saldaña J. Qualitative data analysis: a methods sourcebook. Thousand Oaks, CA: SAGE Publications; 2013.

34. Moos RH, Moos BS. Treated and untreated alcohol-use disorders: course and predictors of remission and relapse. Eval Rev. 2007;31(6):564-84. 
35. Ortiz SN, Espel-Huynh HM, Felonis C, Scharff A. Qualitative perceptions of and preferences for the research process among patients with eating disorders. Int J Eat Disord. 2020;53(1):41-51.

36. Planner C, Bower P, Donnelly A, Gillies K, Turner K, Young B. Trials need participants but not their feedback? A scoping review of published papers on the measurement of participant experience of taking part in clinical trials. Trials. 2019;20(1):381.

37. Priester MA, Browne T, Iachini A, Clone S, DeHart D, Seay KD. Treatment access barriers and disparities among individuals with co-occurring mental health and substance use disorders: an integrative literature review. J Subst Abuse Treat. 2016;61:47-59.

38. Randles D, Tracy JL. Nonverbal displays of shame predict relapse and declining health in recovering alcoholics. Clin Psychol Sci. 2013;1(2):149-55.

39. Robinson KA, Dennison CR, Wayman DM, Pronovost PJ, Needham DM. Systematic review identifies number of strategies important for retaining study participants. J Clin Epidemiol. 2007;60(8):757.e1-757.e19.

40. Rose D. Service user/survivor-led research in mental health: epistemological possibilities. Disabil Soc. 2017;32(6):773-89.

41. Roth RM, Isquith PK, Gioia GA. Behavioral rating inventory of executive function-Adult version. Lutz, FL: Psychological Assessment Resources; 2005.

42. Rubin DB. Inference and missing data. Biometrika. 1976;63(3):581-92.

43. Scott CK. A replicable model for achieving over $90 \%$ follow-up rates in longitudinal studies of substance abusers. Drug Alcohol Depend. 2004;74(1):21-36.

44. Scott CK, White W, Dennis ML. Chronic addiction and recovery management: Implications for clinical practice. Counselor (Deerfield Beach). 2007;8(2):22-7.

45. Sømhovd M, Hagen E, Bergly T, Arnevik EA. The montreal cognitive assessment as a predictor of dropout from residential substance use disorder treatment. Heliyon. 2019;5(3):e01282.

46. Strickland JC, Marks KR, Stoops WW. Researcher perspectives on including people who use drugs in clinical research. Am J Drug Alcohol Abuse. 2020. https://doi.org/ 10.1080/00952990.2020.1790581.
47. Svendsen TS, Bjornestad J, Slyngstad TE, McKay JR, Skaalevik AW, Veseth M, Moltu C, Nesvåg S. "Becoming myself": how participants in a longitudinal substance use disorder recovery study experienced receiving continuous feedback on their results. Subst Abuse Treat Prev Policy. 2020;15:8

48. Svendsen TS, Erga AH, Hagen E, McKay JR, Njå ALM, Årstad J, Nesvåg S. How to maintain high retention rates in long-term research on addiction: a case report. J Soc Work Pract Addict. 2017;17(4):374-87.

49. Tindall L, Smith JA, Flower P, Larkin M. Interpretative phenomenological analysis: theory, method and research. Qual Res Psychol. 2009;6(4):346-7.

50. Topor A, Skogens L, von Greiff N. Building trust and recovery capital: the professionals' helpful practice. Adv Dual Diagn. 2018;11(2):76-87.

51. Tschacher W, Haken H, Kyselo M. Alliance: a common factor of psychotherapy modeled by structural theory. Front Psychol. 2015;6:421.

52. Veseth M, Binder PE, Borg M, Davidson L. Collaborating to stay open and aware: service user involvement in mental health research as an aid in reflexivity. Nord Psychol. 2017;69(4):256-63.

53. Veseth M, Moltu C, Svendsen TS, Nesvåg S, Slyngstad TE, Skaalevik AW, Bjornestad J. A stabilizing and destabilizing social world: close relationships and recovery processes in SUD. J Psychos Rehabil Mental Health. 2019;6(1):93-106.

54. Veseth M, Svendsen TS, Nesvåg S, Moltu C, Davidson L, \& Bjornestad J (In review). "And then the rest happened"-A qualitative exploration of the role meaningful activities play in recovery processes for people with a diagnosis of substance use disorder. Substance Abuse.

55. Wensing M, Elwyn G. Methods for incorporating patients' views in health care. BMJ. 2003;326(7394):877-9.

56. West BT, McCabe SE. Alternative approaches to assessing nonresponse bias in longitudinal survey estimates: an application to substance-use outcomes among young adults in the United States. Am $\mathrm{J}$ Epidemiol. 2017;185(7):591-600.

Publisher's Note Springer Nature remains neutral with regard to jurisdictional claims in published maps and institutional affiliations. 